
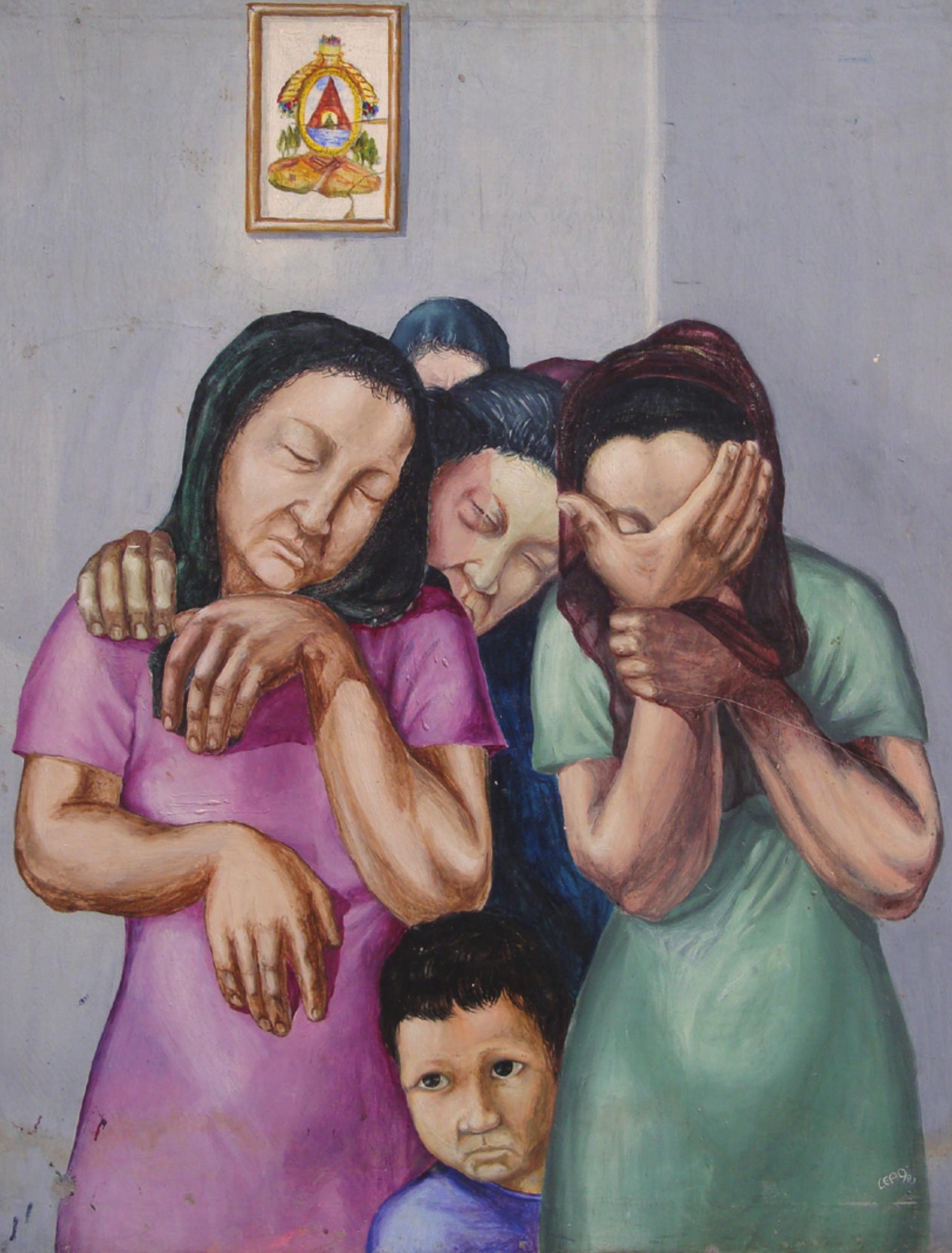


\section{La pobreza en el municipio de La Ceiba según el método de Necesidades Básicas Insatisfechas NBI}

Glordina Poweri Chirinos

\section{INTRODUCCIÓN}

La pobreza es un fenómeno que azota a todos los países del mundo, en Honduras se puede ver la pobreza en todos los departamentos pero si se observa detenidamente esta se esconde en los barrios y colonias más alejadas del casco urbano o centro de las ciudades.

En el municipio de La Ceiba es difícil observar la pobreza a simple vista; para ello es necesario adentrarse a esas comunidades donde la desigualdad de riqueza y oportunidades se pueden palpar.

En vista de la necesidad que existe en la comunidad local de ampliar los conocimientos sobre aspectos relevantes relacionados con la pobreza de este municipio, se presenta el siguiente trabajo de investigación que tiene como objetivo conocer las condiciones de pobreza en el municipio de La Ceiba según el método de Necesidades Básicas Insatisfechas (NBI), para ello se utilizan los datos del último Censo Población y Vivienda realizado en el 2001 y las Encuestas de Hogares, 2006-2007.

Como punto de análisis se tomará en cuenta indicadores del método NBI como el estado o calidad de las viviendas del municipio, disponibilidad de agua potable, saneamiento, educación y hacinamiento.

\section{JUSTIFICACIÓN}

Según investigadores del Postgrado Latinoamericano de Trabajo Social PLATS de la UNAH, los niveles de pobreza y los problemas de empleo condicionan que la gran mayoría de la población no tenga la capacidad para satisfacer necesidades básicas de vivienda, alimentación, saneamiento básico, educación y atención en salud. (PLATS, IDCR, 2007).

La Ceiba es la tercera ciudad del país; aparentemente es una ciudad próspera, sin embargo, una buena parte de la población está en condiciones de pobreza.

Esta investigación pretende conocer mediante el método de Necesidades Básicas Insatisfechas las condiciones de pobreza que se viven en el municipio de La Ceiba departamento de Atlántida.

Esta investigación es un aporte para la sociedad local que permite aclarar las generalidades que existen en relación a la pobreza, también aporta datos para conocimiento nacional y permite a las autoridades que trabajan con programas de Estrategia de Reducción de la Pobreza (ERP) una mejor toma de decisiones y el uso adecuado de fondos.

\section{PLANTEAMIENTO DEL PROBLEMA}

Las características propias del municipio de La Ceiba, como su ubicación estratégica para el comercio y su belleza natural, son incentivos para que su crecimiento poblacional ascienda cada año. El crecimiento poblacional de La Ceiba en comparación con otros municipios de Atlántida, es bastante acelerado debido a la migración de personas o familias completas que buscan una mejor oferta de vida.

En la medida que crece la población también crece la demanda de servicios públicos como agua potable, energía eléctrica, red de alcantarillado y acceso a la educación 
entre otros. Es por ello que esta investigación va dirigida a conocer las condiciones de pobreza en el municipio de La Ceiba según el método de las Necesidades Básicas Insatisfechas (NBI).

\section{OBJETIVOS}

\section{Objetivo General}

Conocer las condiciones de la pobreza en el municipio de La Ceiba según el Método de las Necesidades Básicas Insatisfechas (NBI).

\section{Objetivos Específicos}

- Describir los materiales con que están construidas las viviendas de La Ceiba.

- Identificar los hogares que no cuentan con agua potable en el municipio de La Ceiba según el Censo del 2001.

- Identificar si en las viviendas del municipio de La Ceiba hay hacinamiento.

- Describir el nivel educativo de las personas que conforman los hogares del municipio de La Ceiba.

\section{METODOLOGÍA}

Esta investigación es de carácter cuantitativo. El proceso de consulta se realizó utilizando fuentes secundarias principalmente el Censo Nacional de Población y Vivienda del 2001, las Encuestas Permanentes de Hogares de Propósitos Múltiples, entrevistas con informantes clave y en la Alcaldía Municipal de La Ceiba.

El proceso metodológico que orientó el estudio se diseñó de la siguiente manera:

- Reconocimiento del tema de estudio.

- Delimitación del área de estudio. Se seleccionó a todo el municipio de La Ceiba ya que era necesario conocer y documentar información respecto a la pobreza según el método de las NBI.

- Recopilación y selección de la información. Se recolectó la información de diferentes entidades como DIEM-LC, Secretaria de Educación y otras fuentes; luego se procedió a seleccionar aquella documentación relacionada a la pobreza con aspectos demográficos de La Ceiba. Se trabajó con el programa Redatam con datos del Censo Nacional de Población y Vivienda del 2001 para procesar todos los datos cuantitativos y poder analizarlos con cuidado. También se realizó un desplazamiento a colonias y comunidades rurales para tomar fotografías de las viviendas y anexarlos con el fin de enriquecer la presente investigación.

- Análisis de la información.

- Redacción del informe final.

\section{MUNICIPIO DE LA CEIBA}

\section{- Ubicación}

El departamento de Atlántida, fundado en 1877, se encuentra localizado en el sector septentrional del país. Sus límites son: al norte con el mar Caribe o de las Antillas y el departamento de Islas de la Bahía, al sur con el departamento de Yoro, al este con el departamento de Colón y al oeste con el departamento de Cortés.

Atlántida tiene como cabecera departamental a la ciudad portuaria de La Ceiba, la tercera ciudad más importante del país. La Ceiba es una de las ciudades más atractivas, es una ciudad joven, dinámica, con mucha riqueza y vida natural. Obtiene su nombre, gracias a un árbol llamado Ceiba que con su grande y extensa altura brindaba fresca sombra a los habitantes.

Esta hermosa ciudad puerto fue declarada municipio el 23 de agosto de 1877, durante el gobierno del Dr. Marco Aurelio Soto, siendo el núcleo del emporio bananero y

\section{Figura No. 1. Mapa departamento de Atlántida}

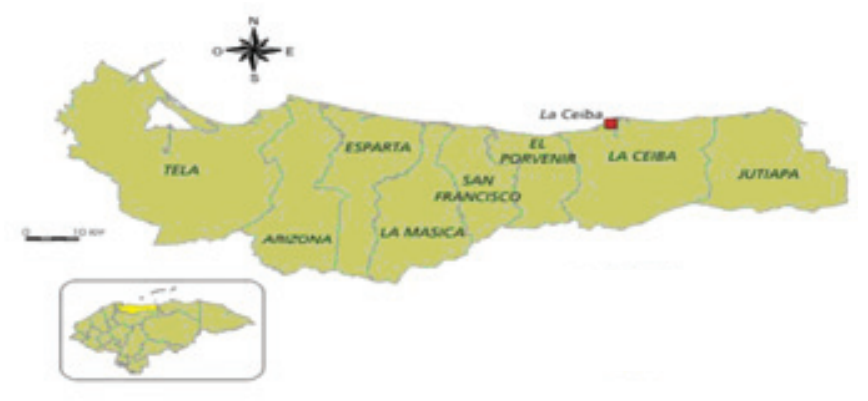

Fuente: www.luventicus.org/mapas 
eje sobre el cual giraba toda la economía regional. Esto llevó al nacimiento de nuevas y más grandes compañías nacionales como la Standard Fruit Company actualmente conocida como Standard Fruit de Honduras que exporta bananos, piñas y cítricos.

\subsection{Datos poblacionales}

A principios de la década de los 90, La Ceiba contaba con una población de 96,226 habitantes (1994). Para el 2001 la población era de 127,590 habitantes de los cuales 61,215 son del sexo masculino y 66,375 del sexo femenino (INE, 2001). Para el 2008 según las proyecciones realizadas por el INE su población ascendía a 175,206 habitantes.

La extensión territorial del municipio de La Ceiba es de $639.45 \mathrm{Kms}^{2}$ con un perímetro urbano $73.22 \mathrm{Kms}^{2}$ (7,322 Hectáreas) y una zona rural que comprende un área de $566.23 \mathrm{Kms}^{2}$. Su extensión representa el $0.6 \%$ en el total del territorio hondureño.

El municipio de La Ceiba cuenta con 14 aldeas, 72 caseríos y 106 barrios y colonias, la densidad poblacional para el año 2008 era de 274 habitantes/ km2; existe un mayor porcentaje de población femenina en el municipio, además cuatro de cada diez personas son niños o jóvenes de 14 años o menos y siete de cada 100 adultos son mayores de 65 años (DIEM, 2008).

Las condiciones de vida de la población han mejorado como lo reflejan los indicadores de esperanza de vida al nacer que es un 70.9 y la tasa de mortalidad infantil que se ha reducido a 17 fallecimientos por cada 1000 nacidos vivos (IDH 2006), sin embargo, existe un sector de la población hacia el cual es necesario derivar acciones urgentes para mejorar su condición de vida y éste es el sector de la tercera edad el cual en estos momentos se encuentra desprotegido. La tasa de crecimiento de la población para el municipio de La Ceiba es del 2.8\% anual, según datos del último censo (INE 2001).

\subsection{Fisiografía del municipio}

La Ceiba se asienta sobre una planicie de sedimentación, de origen marino y aluvial, formando un gran número de abanicos aluviales traslapados. El municipio de La Ceiba está irrigado por los siguientes cursos de agua: río Danto con sus tributarios: La Ausencia, Bulgaria, Calderón, Chiquito y El Padre; río Cangrejal con sus afluentes: Yaruca, Blanco, Viejo y El Padre; y río Bonito, los cuales constituyen los cursos de agua más caudalosos; encontramos además, ríos de menor caudal como, Juana Leandra, Perú, Satuyé, María, Jimerito, Cuyamel, Chiquito, Sambo Creek, Piedras y Ramírez. También existen numerosas quebradas y nacimientos de agua cuyos nombres se detallan a continuación: Buena Vista, La Colorada, La Pita, De Adán, Palmira, Las Mangas, Grande, Los Balicos, De Oro, El Limón, Manga Seca, Quilina, La Muralla, El Pital, Boca Vieja, El Bejuco, Arranca Barba, Susco, Higüero, El Chorro, Tocamacho, El Tigre, La Relumbrosa, La Primavera, entre otros.

En términos generales el clima de La Ceiba, se puede calificar como muy lluvioso, con una distribución regular de las precipitaciones, influenciados por el sistema montañoso predominante de la cordillera Nombre de Dios.

\subsection{Presencia institucional}

En el municipio de La Ceiba hay instituciones que velan y brindan asistencia técnica, financiera y de servicios a la comunidad. Algunos de estos apoyan y trabajan conjuntamente con la municipalidad de La Ceiba en la ejecución de proyectos de infraestructura, saneamiento, producción y capacitación del recurso humano, para que los proyectos gestionados a beneficio de la población se puedan llevar a cabo con mejor eficiencia y a la vez sean sostenibles (cuadro No.1).

\subsection{Aspectos socioeconómicos}

La economía hondureña depende básicamente del sector agropecuario, que en 2002 aportó el 24.6\% del PIB ocupando el primer lugar seguido por la industria textil con un $16.2 \%$. Actualmente la economía del país atraviesa una situación muy difícil por causa de los desajustes financieros, los altos índices de corrupción registrados durante las últimas décadas. La PEA estaba constituida por 2, 569,579 personas, de las cuales 


\section{Cuadro No. 1. Instituciones presentes en La Ceiba}

\begin{tabular}{|l|l|}
\hline \multicolumn{1}{|c|}{ Institución } & \multicolumn{1}{c}{ Descripción } \\
\hline SANAA & $\begin{array}{l}\text { Servicio de agua potable y alcan- } \\
\text { tarillado }\end{array}$ \\
\hline SOPTRAVI & $\begin{array}{l}\text { Atención a vías de transporte y } \\
\text { aeropuerto }\end{array}$ \\
\hline INFOP & Formación ocupacional \\
\hline ENEE & Servicio de energía eléctrica \\
\hline COHDEFOR & Protección del bosque \\
\hline SAG & $\begin{array}{l}\text { Asistencia técnica y financiera al } \\
\text { sector productivo }\end{array}$ \\
\hline HONDUTEL & Servicio de telefonía \\
\hline Secretaria de TURISMO/ & Apoyo al turismo del municipio \\
\hline Proyecto Costero & Desarrollo Comunitario, Agua \\
\hline Cooperación Española & Potable \\
\hline Secretaria de Recursos & $\begin{array}{l}\text { Apoyo a la protección de los recur- } \\
\text { sos naturales }\end{array}$ \\
\hline Pastoral Social /Iglesia & Desarrollo Comunitario \\
\hline Católica & Apoyo al comercio \\
\hline Cámara de Comercio & Promoción y apoyo al turismo \\
\hline Cámara de Turismo &
\end{tabular}

Fuente: Diagnóstico de La Ceiba, 2006

1,695,943 (66\%) eran hombres y 873,636 (34\%) mujeres (EPHPM2007).

Como se observa en el cuadro No. 2, las principales ocupaciones desarrolladas por los habitantes del municipio de La Ceiba se distribuyen de la siguiente manera: los trabajadores de la industria textil, albañilería, mecánica y electricidad, representan un porcentaje alto de $21.84 \%$ indicando que la mayoría de la población se basa en esta actividad para el sustento y desarrollo de la economía local, seguido por el turismo (venta de servicios) con un porcentaje significativo de $14,66 \%$, también se observa que el comercio local ha ido en crecimiento ya que es una de las principales ocupaciones que se lleva a cabo en esta ciudad, asimismo está la agricultura, ganadería y trabajos agropecuarios en porcentajes menores. En baja escala encontramos los operadores de carga, almacenaje con un 3.43\% así como trabajadores en el área de gráfica, química, alimentos y bebidas, cuero, tabaco y cerámica con $2.69 \%$, no se incluye las remesas familiares de hon-

\section{Cuadro No. 2. Principales ocupaciones económicas} en La Ceiba

\begin{tabular}{|l|c|c|}
\hline Categorías & Casos & $\%$ \\
\hline $\begin{array}{l}\text { 0. Profesionales, técnicos y personas } \\
\text { en profesiones afines }\end{array}$ & 5,003 & $13.15 \%$ \\
\hline $\begin{array}{l}\text { 1. Directores, gerentes y administra- } \\
\text { dores generales }\end{array}$ & 1,549 & $4.07 \%$ \\
\hline $\begin{array}{l}\text { 2. Empleados de oficinas del estado, } \\
\text { organismos autónomos y empresa } \\
\text { privada }\end{array}$ & 2,111 & $5.55 \%$ \\
\hline \begin{tabular}{l} 
3. Comerciantes y vendedores \\
\hline 4. Agricultores, ganaderos y trabaja- \\
dores agropecuarios
\end{tabular} & 2,829 & $7.43 \%$ \\
\hline $\begin{array}{l}\text { 5. Conductores de medios de trans- } \\
\text { porte y personas en ocupaciones } \\
\text { afines }\end{array}$ & 2,359 & $6.20 \%$ \\
\hline $\begin{array}{l}\text { 6. Trabajadores de la industria textil, } \\
\text { albañilería,mecánica, electricidad, } \\
\text { etc. }\end{array}$ & 8,310 & $21.84 \%$ \\
\hline $\begin{array}{l}\text { 7. Trabajadores del área gráfica, quí- } \\
\text { mica, alimentos y bebidas, cuero, } \\
\text { tabaco, cerámica }\end{array}$ & 1,024 & $2.69 \%$ \\
\hline $\begin{array}{l}\text { 8. Operadores de carga, almacenaje } \\
\text { y estiba }\end{array}$ & 1,305 & $3.43 \%$ \\
\hline \begin{tabular}{l} 
9. Ocupación de los servicios \\
\hline 10. No especificas
\end{tabular} & 5,579 & $14.66 \%$ \\
\hline \begin{tabular}{l} 
Total \\
\hline
\end{tabular} & 2,435 & $6.40 \%$ \\
\hline
\end{tabular}

Fuente: Elaboración propia en base al XVI Censo Nacional Población y vivienda 2001

dureños en el extranjero, que representan una importante fuente de ingreso para varias familias.

VII. LA POBREZA EN EL MUNICIPIO DE LA CEIBA SEGÚN EL MÉTODO DE NECESIDADES BÁSICAS INSATISFECHAS (NBI).

La pobreza es un fenómeno social que tiene lugar cuando el individuo, la familia y las masas tienen carencia y estrechez en necesidades básicas tales como: salud, educación, vivienda, alimentación y oportunidades (Román, Rafael, 1997); es un fenómeno complejo y multidimensional, razón por la cual existen múltiples definiciones y maneras de medirla.

La pobreza es hambre. La pobreza es falta de techo bajo el cual resguardarse. La pobreza es estar enfermo y 
no poder ser atendido por un médico. La pobreza es no poder ir a la escuela y no saber leer. La pobreza es no tener trabajo, tener miedo al futuro y vivir día a día. La pobreza es perder a un hijo debido a enfermedades relacionadas con el agua impura. La pobreza es impotencia, falta de representación y libertad.

\subsection{La pobreza en Honduras y en el municipio de La Ceiba}

Honduras es considerado el país más pobre de la región después de Haití y Nicaragua. El fenómeno de pobreza se acentúa en el área rural, estas son las áreas que presentan mayor limitación en cuanto a la cobertura y calidad de los servicios sociales. En el marco de reducir la pobreza el gobierno ha tomado planes de acción a mediano y largo plazo y por ende se han creado políticas de Estado tales como la Estrategia para la Reducción de la Pobreza y los Objetivos y Metas del Milenio.

Con la ERP el país ha priorizado seis áreas programáticas, entre ellas, una con la que se pretende reducir la pobreza en zonas rurales. Esta línea de acción tiene como objetivo, mejorar el acceso equitativo, seguro y sostenible a factores productivos (tierra, capital, recursos humanos etc.) que incluye la generación de empleo e ingresos para la población hondureña.

En el municipio de La Ceiba se maneja fondos ERP mediante transferencias que el gobierno hace a la $\mathrm{Mu}$ nicipalidad, la cual se encarga directamente de apoyar al sector rural y pobre del municipio con proyectos productivos, sociales y de gobernabilidad con el fin de reducir los niveles de pobreza en este municipio. Aún contando con instrumentos valiosos como la ERP, la pobreza y la pobreza extrema sigue siendo más aguda en las zonas rurales que en el casco urbano aunque esta última también se ve afectada por los incrementos de la canasta básica.

La Encuesta de Hogares realizada por el Instituto Nacional de Estadística (INE), entre los meses de mayo a julio de 2007, destaca que la pobreza en Honduras disminuyó del $61.8 \%$ a un $60.2 \%$. Pero a pesar de esa disminución de 1.6 puntos porcentuales; según el PNUD (2005) en Honduras hay aproximadamente 1, 500,000 personas que sobreviven con menos de un dólar diario. No tienen suficiente dinero para comprar la comida y vestimenta que necesitan. Muchos no tienen acceso al agua limpia ni a un refugio.

Según el informe de la ERP (2001) las causas de la pobreza en Honduras, son por problemas vinculados al lento crecimiento económico y al bajo ingreso per cápita; la desigual distribución del ingreso y de los factores de la producción; el bajo nivel de escolaridad; y la baja productividad del empleo. Además, la pobreza se vincula con factores como: la presión demográfica y sus efectos sobre los recursos naturales; el bajo nivel de participación de los pobres; el deterioro de los valores culturales y las debilidades de los gobiernos locales.

Aunque la incidencia de la pobreza es mayor en las áreas rurales (66.4\% de los hogares), en el área urbana alcanza más de la mitad (55.4\%) (ERP 2001). La pobreza tanto relativa y extrema se concentra en la zona rural del país, pero la zona urbana también tiene sus índices de pobreza. Dos de cada tres hogares en el país son pobres.

Para conocer la pobreza de La Ceiba según el método de las Necesidades Básicas Insatisfechas se detallan las condiciones de las viviendas que incluye: techos, paredes y pisos, además del hacinamiento, la educación y acceso de agua potable; no se toma en cuenta la capacidad de subsistencia ya que la fuente principal en esta investigación es el Censo de Población y Viviendas del 2001 y no tiene datos relacionados a ese aspecto.

\subsection{La características de las vivienda en el muni-} cipio de La Ceiba

El municipio de La Ceiba según el censo del año 2001 cuenta con 33,997 viviendas ocupadas de las cuales el 40\% tienen Necesidades Básicas Insatisfechas. En cifras relativas refleja menos de la mitad de la población pero si este porcentaje lo convertimos en cifras absolutas es alarmante ya que se observará un número alto de viviendas en el municipio con NBI. A partir del censo del 2001 se estableció que la tasa de crecimiento de viviendas de La Ceiba era de $4.7 \%$ anual (INE, 2001). 
Según estimaciones del Departamento de Investigación y Estadística Municipal de La Ceiba (DIEM) la demanda de viviendas para este municipio en el 2008 es de 44,988 viviendas para el área urbana y 3,065 para el área rural, para los próximos años aumentará la demanda de los mismos, provocado por el mismo crecimiento poblacional (cuadro No. 3).

La alta demanda de viviendas es por la carencia de una estructura y de un sistema institucional tanto central como descentralizado capaz de asumir el reto del déficit habitacional, que según estudios realizados por Aradhos (2007) se encuentra en un 37\%, afectando a muchas familias ceibeñas. El desarrollo de proyectos habitacionales se ha visto limitado por falta de tierra urbanizable, esto obliga a que en esta ciudad se recurra a la construcción de soluciones habitacionales carentes de infraestructura social y sanitaria adecuada en lugares marginales de la ciudad.

En términos generales, los programas de vivienda de parte de instituciones dedicadas a solucionar este

\section{Cuadro No. 3. Proyección de las necesidades de vivienda rural y urbana del municipio de La Ceiba: 2002 al 2015}

\begin{tabular}{|l|l|c|c|}
\hline Año & Total & $\begin{array}{c}\text { Vivienda Rural } \\
(\mathbf{6 . 3 8})\end{array}$ & $\begin{array}{c}\text { Vivienda Urbana } \\
(\mathbf{9 3 . 6 2 \% )}\end{array}$ \\
\hline 2002 & 36,395 & 2,322 & 34,073 \\
\hline 2003 & 38,146 & 2,434 & 35,712 \\
\hline 2004 & 39,982 & 2,540 & 37,278 \\
\hline 2005 & 41,906 & 2,662 & 39,072 \\
\hline 2006 & 43,923 & 2,790 & 40,952 \\
\hline 2007 & 46,037 & 2,924 & 42,923 \\
\hline 2008 & 48,252 & 3,065 & 44,988 \\
\hline 2009 & 50,574 & 3,212 & 47,153 \\
\hline 2010 & 53,008 & 3,367 & 49,422 \\
\hline 2011 & 55,559 & 2,790 & 40,952 \\
\hline 2012 & 58,233 & 2,924 & 42,923 \\
\hline 2013 & 61,035 & 3,065 & 44,988 \\
\hline 2014 & 63,972 & 3,212 & 47,153 \\
\hline 2015 & 67,051 & 3,367 & 49,422 \\
\hline
\end{tabular}

Fuente: Cálculo realizado por el DIEM-LC en base a una Tasa de Crecimiento de la Vivienda del $4.7 \%$ Anual, la cual fue establecida por el INE de los Resultados obtenidos en el Censo nacional del 2001 problema son insuficientes, por un lado debido al alto costo de viviendas y a la poca capacidad adquisitiva de gran parte de la población.

7.3 Materiales de construcción de las viviendas en el municipio de La Ceiba

\subsubsection{Material del techo en las viviendas de La Ceiba}

Como lo refleja el cuadro No. 4 los techos de las viviendas en el municipio de La Ceiba están construidos en su mayoría de láminas de zinc representando el 80.13\% seguido por lámina de asbesto con un $11.93 \%$. También existen 482 viviendas que tienen techo de paja o palma representando el $1.42 \%$ y 362 construidas de material de desecho que representan el 1.07\% respectivamente.

En La Ceiba los materiales de construcción que han utilizado han ido variando con el tiempo, dependiendo de las necesidades y disponibilidad de recursos de la población. En el caso del techo de las viviendas, en La Ceiba se puede apreciar la existencia de una variedad de materiales, no obstante el predominante es el de lámina de zinc. Este tipo tiene la característica de que su costo es menor a otros materiales utilizados para este fin y son manejables porque son más livianos que otros materiales utilizados. Aunque presenta algunas desventajas: en verano con facilidad absorbe el calor, volviendo las viviendas muy calurosas mientras que en épocas de invierno se vuelve frío, por lo que este no brinda la protección adecuada y

\section{Cuadro No. 4. Materiales de construcción de los techos de las viviendas en La Ceiba}

\begin{tabular}{|l|c|c|}
\hline \multicolumn{1}{|c|}{ Categorías } & Casos & $\%$ \\
\hline 1. Teja de Barro & 191 & $.56 \%$ \\
\hline 2. Lámina de asbesto & 4,048 & $11.93 \%$ \\
\hline 3. Lámina de zinc & 27,195 & $80.13 \%$ \\
\hline 4. Concreto & 858 & $2.53 \%$ \\
\hline 5. Paja, palma o similar & 482 & $1.42 \%$ \\
\hline 6. Material de desecho & 362 & $1.07 \%$ \\
\hline 7. Otro & 804 & $2.37 \%$ \\
\hline Total & 33,940 & $100.00 \%$ \\
\hline
\end{tabular}

Fuente: Elaboración propia en base al XVI Censo Nacional Población y vivienda 2001 
necesaria. Además en tiempos de tempestades el zinc se vuelve vulnerable ya que se puede desprender de la estructura. Otro material que se utiliza con frecuencia es el asbesto ya que es un mejor aislante de la temperatura, aunque tienen desventajas: son muy frágiles, su costo es más elevado y requiere de mano de obra calificada para su instalación.

\subsubsection{Material de las paredes en La Ceiba}

Según lo que indica el cuadro No. 5, los materiales de construcción más utilizados son el bloque de cemento con un $75.28 \%$, seguido por la madera con $17.24 \%$ También se observa que hay un $1.22 \%$ o sea 415 viviendas que tienen las paredes construidas de bahareque otros de adobe y materiales de desecho entre otros.

Existen casas que sus paredes son de bloque, otras de madera, bahareque, otras tienen las paredes construidas de diferentes materiales. Pero también existen casas que sus paredes son muy débiles, construidas con desperdicios de lámina de zinc, plásticos, cartón etc. El material predominante en las paredes es el bloque de cemento, el cual se puede adquirir en cualquier bloquera de la ciudad. Pese a que el bloque es un material durable y barato, presenta algunas desventajas:

7.3.3 Material del piso de la vivienda en la ciudad de La Ceiba

Como lo muestra el cuadro No. 6, el 60.10\% de los pisos de las viviendas de La Ceiba tienen plancha de cemento o sea 16,740 viviendas, seguido por piso de

\section{Cuadro No. 5 Materiales de construcción de las pa-} redes de las viviendas en el Municipio de La Ceiba

\begin{tabular}{|l|c|c|}
\hline \multicolumn{1}{|c|}{ Categorías } & Casos & $\%$ \\
\hline 1. Ladrillo rafón & 308 & $0.91 \%$ \\
\hline 2. Piedra rajada o cantera & 116 & $0.34 \%$ \\
\hline 3. Bloque de cemento & 25,551 & $75.28 \%$ \\
\hline 4. Adobe & 390 & $1.15 \%$ \\
\hline 5. Madera & 5,851 & $17.24 \%$ \\
\hline 6. Bahareque & 415 & $1.22 \%$ \\
\hline 7. Palo o caña & 151 & $0.44 \%$ \\
\hline 8. Material de desecho & 362 & $1.07 \%$ \\
\hline 9. Otro & 796 & $2.35 \%$ \\
\hline Total & 33,940 & $100.00 \%$ \\
\hline
\end{tabular}

Fuente: Elaboración propia en base al XVI Censo Nacional Población y vivienda 2001

tierra que representa el $11.49 \%$ que en cifras absolutas son 3,199 viviendas significando esto que estas viviendas pueden ser insalubres para las personas que las habitan sobre todo para los niños y niñas.

En La Ceiba las viviendas tienen características variadas en cuanto al tamaño, forma y materiales utilizados para su construcción. La mayoría de las personas cuentan con una casa adecuada, sin embargo, no reúne las condiciones óptimas debido a que en su construcción utilizan materiales como desperdicios o desechos, bahareque, adobe que si tienen la ventaja de brindar un techo, una pared o piso a la persona pero no reúne las condiciones necesarias para mejorar el nivel y calidad de vida de la población.

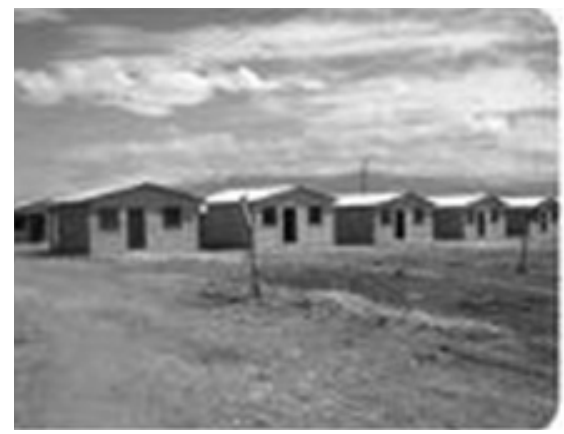

Vista de viviendas con techo de zinc en la col. Sutrasfco

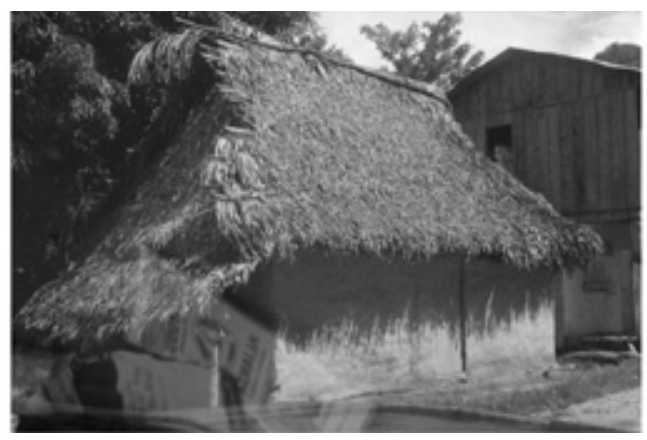

Vista de vivienda con techo de paja en la comunidad de El Pital cuenca del Río Cangrejal.

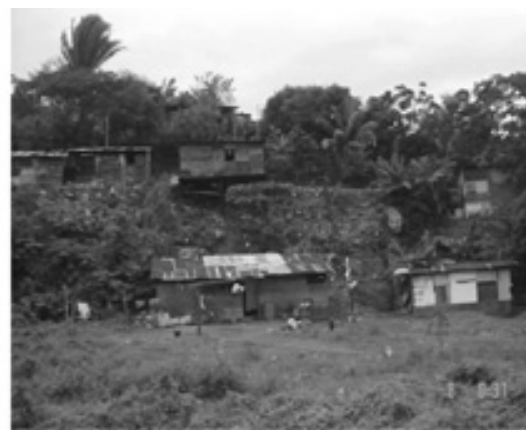

Vista de las viviendas hechas de desechos del asentamiento aguas abajo del relleno Los Laureles. 
Cuadro No. 6. Materiales de construcción de pisos de las viviendas en La Ceiba

\begin{tabular}{|l|c|c|}
\hline \multicolumn{1}{|c|}{ Categorías } & Casos & $\%$ \\
\hline 1. Tierra & 3,199 & $11.49 \%$ \\
\hline 2. Plancha de cemento & 16,740 & $60.10 \%$ \\
\hline 3. Madera & 1,777 & $6.38 \%$ \\
\hline 4. Ladrillo cemento & 2,874 & $10.32 \%$ \\
\hline 5. Ladrillo Terrazo & 1,753 & $6.29 \%$ \\
\hline 6. Ladrillo Barro & 22 & $0.08 \%$ \\
\hline 7. Cerámica & 983 & $3.53 \%$ \\
\hline 8. Otro & 505 & $1.81 \%$ \\
\hline Total & $\mathbf{2 7 , 8 5 3}$ & $\mathbf{1 0 0 . 0 0} \%$ \\
\hline
\end{tabular}

Fuente: Elaboración propia en base al XVI Censo Nacional Población y vivienda 2001

\subsection{Acceso a servicios básicos en la ciudad de La} Ceiba

\subsubsection{Acceso a agua potable en La Ceiba}

El agua es un recurso abundante en el municipio de La Ceiba, ya que existe gran cantidad de ríos, quebradas y nacimientos, los que están diseminados en toda la extensión del territorio, de toda esa cantidad en fuentes de agua solamente los ríos Bonito y Danto, producen agua que es apta para consumo humano.

En la actualidad existen problemas considerables en cuanto al suministro de agua potable, a tal grado que en la estación seca y aun en la estación lluviosa, se debe recurrir a programas de racionamiento, debido a que se ha estado haciendo un inadecuado uso de los recursos naturales, por lo que el caudal de los ríos que suministran el vital líquido, está disminuyendo de forma alarmante

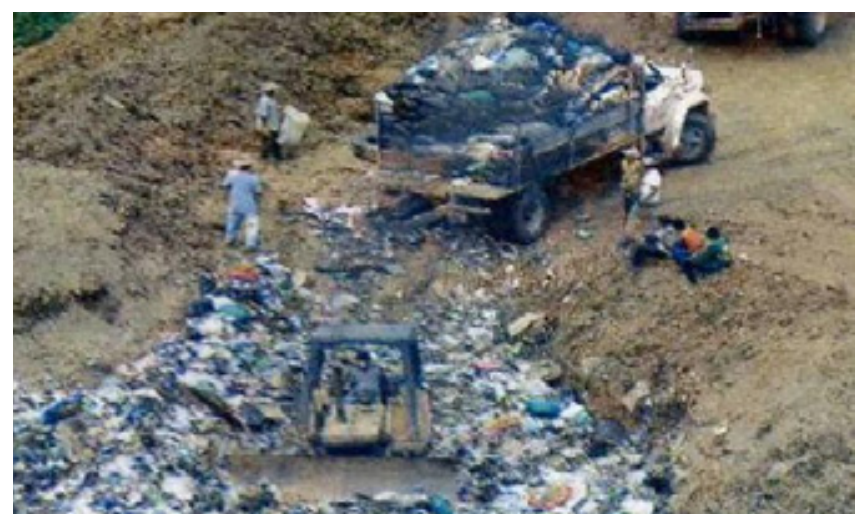

Vista del relleno sanitario a excepción del río Bonito, que se encuentra en buen estado de conservación especialmente en la zona donde se ubica tubería de agua potable, cuya área esta debidamente decretada y delimitada, por lo que no existe intervención humana en su entorno.

La oferta actual del servicio de agua potable es insuficiente para satisfacer la demanda de toda la población de esta ciudad de La Ceiba.

Según muestra el cuadro No. 7 y gráfico No. 1 el $83.48 \%$ de las viviendas en el municipio están conectadas al sistema público o privado, el $56.46 \%$ cuenta con tubería dentro de la casa y el $31.59 \%$ fuera de ella como lo muestra el gráfico No. 1 a la vez se observa un porcentaje de $5.94 \%$ de las viviendas que extraen el agua de pozo malacate, seguido por pozo con bomba que es un poco

\section{Cuadro No. 7. Formas de abastecimiento de agua} en La Ceiba

\begin{tabular}{|l|c|c|}
\hline \multicolumn{1}{|c|}{ Categorías } & Casos & $\%$ \\
\hline $\begin{array}{l}\text { 1. De tubería del sistema público/ } \\
\text { privado }\end{array}$ & 23,253 & $83.48 \%$ \\
\hline 2. De pozo malacate & 1,655 & $5.94 \%$ \\
\hline 3. De pozo con bomba & 945 & $3.39 \%$ \\
\hline 4. De vertiente, río & 818 & $2.94 \%$ \\
\hline 5. De lago o laguna & 11 & $.04 \%$ \\
\hline 6. De vendedor o repartidor & 103 & $.37 \%$ \\
\hline 7. Otro & 1,068 & $3.83 \%$ \\
\hline Total & 27,853 & $100.00 \%$ \\
\hline
\end{tabular}

Fuente: Elaboración propia en base al XVI Censo Nacional Población y vivienda 2001

\section{Gráfico No. 1. Ubicación de tubería de agua potable en La Ceiba}

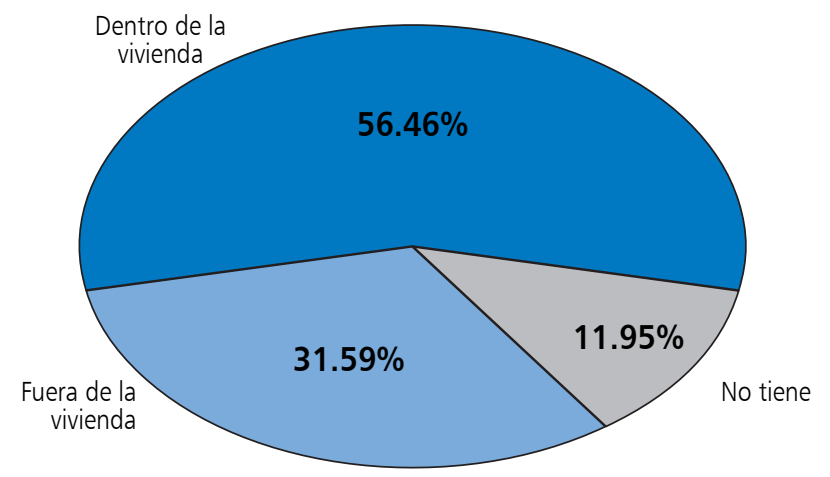

Fuente: Elaboración propia en base al XVI Censo Nacional Población y Vivienda 2001 
más sofisticado que la anterior. Además el 2.94\% de las viviendas se abastecen de agua del río significando que no tienen este líquido vital en sus hogares.

El agua es un líquido vital para la sobrevivencia humana, el 11.95\% de la población de La Ceiba no está conectado a la tubería de agua, y por ende tiene que buscar la manera de obtener este elemento vital de diferentes formas. Este es un problema que afecta una parte importante de la población y aunque un porcentaje alto está conectado a la tubería pública estas personas no reciben en forma continua este líquido, en la mayor parte del tiempo hay racionamiento de agua. Aquellas familias que no pueden costear este servicio no tienen otra opción más que acarrear agua de los ríos y los pozos o no contar con este liquido vital.

\subsubsection{Saneamiento básico}

\section{- Relleno sanitario}

Según información del departamento municipal de servicios públicos el tren de aseo es el responsable de las actividades de aseo y limpieza de la ciudad, este servicio clasifica la basura en: domiciliaria para barrios y colonias, restaurantes y supermercados, hospitales y clínicas, instituciones gubernamentales, comercial e industrial y mercado, sin embargo, no cubre todas las viviendas del sector.

Para cada una de estas áreas existe un calendario de recolección, el servicio cuenta con 37 vehículos con los que se recogen 120 toneladas de basura, esta es llevada al relleno sanitario localizado en la colonia Los Laureles ubicado sobre la margen derecha del Río Cangrejal en su intersección con la carretera a Trujillo. La operación del sitio ha sido contratada con la firma WPP Continental de Honduras, S.A. La Municipalidad paga a WPP US\$ 12 por tonelada de residuos que recibe para su disposición final.

Según el Censo de Población del 2001, del 100\% de la basura generada en este municipio, el 69.16\% es recogida por el tren de aseo, un $25.98 \%$ la queman o entierran y el $2.88 \%$ que en cifras absolutas son 803 casos la tira a

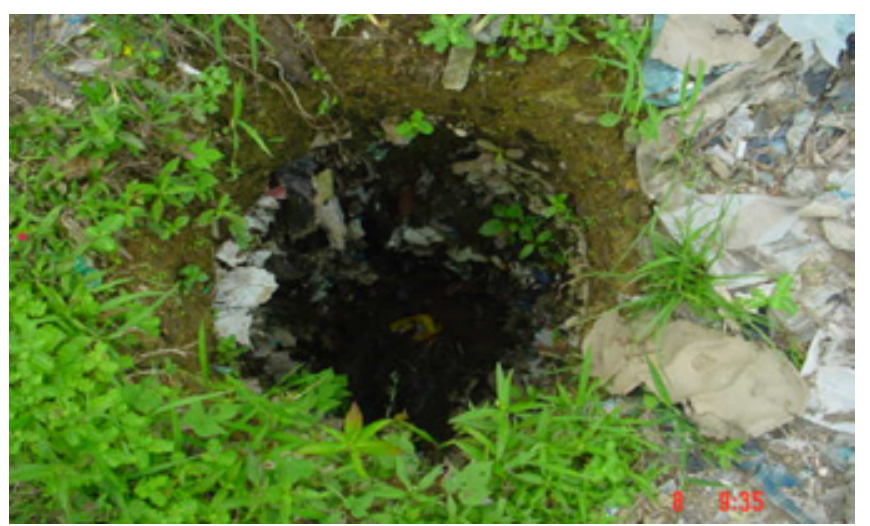

Vista de una excavación practicada al norte del relleno, donde se aprecian los residuos enterrados en la zona que no se ha practicado cierre definitivo.

\section{Cuadro No. 8. Formas de eliminación de la basura en La Ceiba}

\begin{tabular}{|l|c|c|}
\hline \multicolumn{1}{|c|}{ Categorías } & Casos & $\%$ \\
\hline 1. La tira a la calle & 803 & $2.88 \%$ \\
\hline 2. La recoge el carro de la basura & 19,264 & $69.16 \%$ \\
\hline 3. La lleva al depósito & 332 & $1.19 \%$ \\
\hline 4. La quema o entierra & 7,237 & $25.98 \%$ \\
\hline 5. Paga a particulares & 95 & $.34 \%$ \\
\hline 6. Otro & 122 & $.44 \%$ \\
\hline Total & 27,853 & $100.00 \%$ \\
\hline
\end{tabular}

Fuente: Elaboración propia en base al XVI Censo Nacional Población y vivienda 2001

la calle (Cuadro No. 8). En La Ceiba el manejo de los desechos se ha vuelto un reto.

Con la limpieza de la ciudad se está contribuyendo a la conservación del medio ambiente y prevención de enfermedades ocasionadas por la presencia de elementos contaminantes en la comunidad, el municipio de La Ceiba cuenta con un botadero de basura denominado Los Laureles, pero este es insuficiente para responder a la demanda del municipio debido al incremento poblacional.

El relleno sanitario es otro de los problemas de este municipio, según información de servicios públicos de la municipalidad de La Ceiba actualmente el relleno de Los Laureles está a punto de colapsar y es necesario encontrar otro lugar con urgencia donde trasladar el relleno. Por lo que la municipalidad desde hace algún tiempo realiza gestiones para la obtención de fondos con miras a construir 
un nuevo relleno que pueda abastecer no sólo la demanda actual sino una proyección de crecimiento poblacional de 15-20 años. No obstante, aquellas personas que no se les brinda el servicio de recolección de basura, ya sea porque no pueden pagarlo o porque por sus casas no pasa el servicio, solventan esta necesidad quemando la basura, enterrándola o tirándola a la calle, como consecuencia de ello contaminan la ciudad, afectando la salud de la población, sobre todo la de los niños y niñas.

- Alcantarillado sanitario

En cuanto al alcantarillado sanitario la Municipalidad de La Ceiba tiene a su cargo esta tarea, actualmente la Oficina Municipal de Agua y Saneamiento (OMASAN) se encarga directamente de esta labor. Se cuenta con una laguna de oxidación que sirve para tratamiento de aguas negras de orden doméstico en el sector oeste de la ciudad, en el sector este recientemente se puso en funcionamiento un complejo de lagunas de oxidación que son utilizadas para tratamiento de aguas negras domésticas en este sector.

Las aguas residuales son descargadas en esteros y canales los que actúan como cuerpos receptores (sin tratamiento previo). En relación a lo anterior, en el futuro cercano, el sistema de aguas lluvias servirá para la descarga de las aguas residuales domésticas lo que permitirá evitar la contaminación de esteros y canales.

El cuadro No. 9 refleja que de las 28, 174 viviendas encuestadas en el 2001 el $41.83 \%$ cuenta con pozo séptico éstos deberían ser supervisados con periodicidad para asegurar que sean construidos y manejados de la forma más adecuada, se observa que el 30.25\% está co-

\section{Cuadro No. 9. Servicio sanitario en La Ceiba}

\begin{tabular}{|l|c|c|}
\hline \multicolumn{1}{|c|}{ Categorías } & Casos & $\%$ \\
\hline 1. Inodoro red alcantarillado & 8,523 & $30.25 \%$ \\
\hline 2. Inodoro pozo séptico & 11,784 & $41.83 \%$ \\
\hline 3. Inodoro con descarga a río & 263 & $.93 \%$ \\
\hline 4. Letrina de pozo simple & 6,054 & $21.49 \%$ \\
\hline 5. No tiene & 1,550 & $5.50 \%$ \\
\hline Total & 28,174 & $100.00 \%$ \\
\hline
\end{tabular}

Fuente: Elaboración propia en base al XVI Censo Nacional Población y vivienda 2001 nectado a la red de alcantarillado sanitario, el 21.49\% de las viviendas cuenta apenas con letrina de pozo simple y un porcentaje alto $5.50 \%$ de viviendas que no tienen servicio sanitario y en consecuencia hacen sus necesidades al aire libre contaminando el medio ambiente y afectando la salud.

Este servicio resulta también ineficiente en esta ciudad ya que la mayor parte de la población tiene que construir pozo séptico porque el servicio de alcantarillado no ha podido satisfacer la demanda actual y además la mayor parte de los hogares no cuentan con recursos suficientes para adquirirlo.

En conclusión el acceso al saneamiento básico es un problema que afecta a una gran parte de la población porque además de un manejo ineficiente de los servicios no está al alcance de todos debido a los altos costos.

\section{- Energía eléctrica}

Para satisfacer la demanda de energía eléctrica existente en la ciudad de La Ceiba la ENEE que cuenta con un sistema de distribución eléctrica que se alimenta de una subestación en el sector oeste de la ciudad (energía térmica).

Debido a la demanda de energía en la actualidad, se ha considerado la ejecución del proyecto hidroeléctrico El Cangrejal, y se espera que genere 50 mgw de energía. Con la puesta en funcionamiento de este proyecto se pretende satisfacer adecuadamente la demanda energética del municipio de La Ceiba y el Bajo Aguán.

\section{Cuadro No. 10. Formas de uso de energia en La Ceiba}

\begin{tabular}{|l|c|c|}
\hline \multicolumn{1}{|c|}{ Categorías } & Casos & $\%$ \\
\hline 1. Electricidad público & 24,118 & $86.59 \%$ \\
\hline 2. Electricidad privado & 142 & $0.51 \%$ \\
\hline $\begin{array}{l}\text { 3. Electricidad de motor } \\
\text { propio }\end{array}$ & 18 & $0.06 \%$ \\
\hline 4. Candil lámpara de gas & 1,649 & $5.92 \%$ \\
\hline 5. Vela & 1,714 & $6.15 \%$ \\
\hline 6. Ocote & 47 & $0.17 \%$ \\
\hline 7. Panel solar & 7 & $0.03 \%$ \\
\hline 8. Otro & 158 & $0.57 \%$ \\
\hline Total & 27,853 & $100.00 \%$ \\
\hline
\end{tabular}

Fuente: Elaboración propia en base al XVI Censo Nacional Población y Vivienda 2001 
El 86.59\% (Cuadro No. 10) de las viviendas de este municipio está conectado al alumbrado público, el $6.15 \%$ utiliza la vela para alumbrarse seguido por candil o lámpara de gas con un 5,92\%. (Censo 2001).

En cuanto a energía eléctrica la mayor parte de la población está conectada al servicio que presta la ENEE, pero, en los últimos años su costo ha aumentado, y el efecto del ajuste de combustible que según el programa del gobierno beneficia a los más pobres recae directamente sobre la clase media de la población provocando un descenso en la calidad de vida.

En general el acceso a los servicios básicos como agua potable, relleno sanitario, alcantarillado sanitario y energía eléctrica no reúnen las condiciones necesarias y no asiste a toda la población debido a los altos costos.

\subsection{Hacinamiento en la ciudad de La Ceiba}

El hacinamiento es definido como la existencia de más de 3 personas por pieza en una vivienda. Se puede decir que el $42.82 \%$ de los hogares ceibeños presenta dicha situación, es decir, 11,926 viviendas cuentan con 3 ó más personas por cuarto (Gráfico No. 2).

\subsection{Educación en el municipio de La Ceiba}

A nivel de país más del 10\% de la población de 6 a 24 años de edad aún está sin educación, es decir 10 de cada 100 personas son analfabetas.

\section{Gráfico No. 2. Hogares que cuentan con hacinamiento}

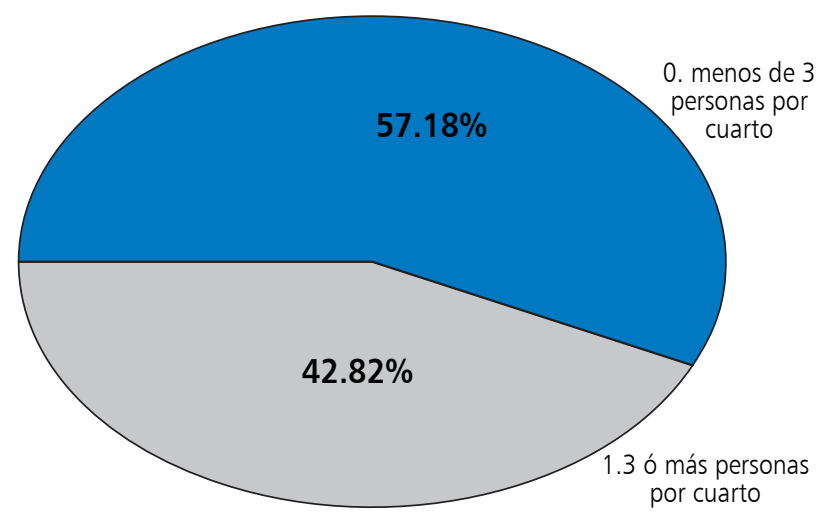

Fuente: Elaboración propia en base al XVI Censo Nacional Población y Vivienda 2001
En esta última década el gobierno de Honduras se comprometió a mejorar la educación, los logros son todavía modestos; sin embargo, el avance fue mayor debido a la puesta en funcionamiento con apoyo de la cooperación externa de varios proyectos de educación de amplia cobertura para jóvenes y adultos, entre ellos se destacan el proyecto EDUCATODOS y PRALEBAH. El proyecto Educatodos (EFA sus siglas en ingles) ha realizado caravanas en todos los departamentos y municipios del país. Esta caravana llegó a La Ceiba destacándose como la segunda en alcanzar las metas EFA en un 75\%, y considerada el municipio más alfabeta de toda Honduras.

Según el Censo de Población y Vivienda 2001, en el municipio de La Ceiba el $87.55 \%$ de la población sabe leer y escribir y el $12.45 \%$ no sabe (INE, 2001), este porcentaje representa 13,869 personas en este municipio que son analfabetas representando la décima parte de la población de La Ceiba y esto es preocupante porque La Ceiba es la tercera ciudad de Honduras (Cuadro No.11, Gráfico No.3).

\section{Cuadro No. 11. Casos de personas que saben leer y escribir en el nunicipio de La Ceiba}

\begin{tabular}{|c|c|c|}
\hline Categorías & Casos & $\%$ \\
\hline 1. Si & 97,504 & $87.55 \%$ \\
\hline 2. No & 13,869 & $12.45 \%$ \\
\hline Total & 111,373 & $100.00 \%$ \\
\hline
\end{tabular}

Fuente: Elaboración propia en base al XVI Censo Nacional Población y Vivienda 2001

\section{Gráfico No. 3. Personas que saben leer y escribir en el municipio de La Ceiba}

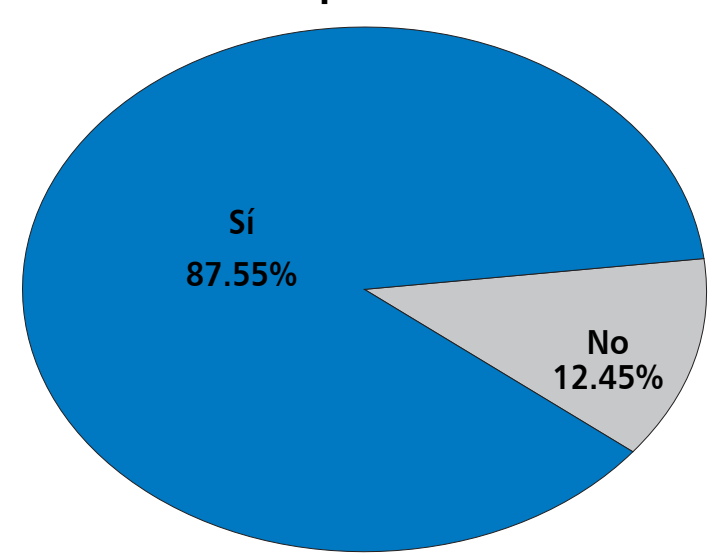

Fuente: Elaboración propia en base al XVI Censo Nacional Población y Vivienda 2001 
Según investigación realizada, La Ceiba está implementando otra medida para minimizar el porcentaje de analfabetismo mediante el programa YO SI PUEDO coordinado por los maestros y con el apoyo del gobierno cubano, este programa pretende alfabetizar a personas de todas las edades con el propósito que puedan aprender.

La pobreza se traduce en las grandes diferencias existentes entre los pueblos y sociedades, en relación al acceso a bienes y servicios básicos, y es consecuencia de los procesos económicos que, con diferentes resultados, se han experimentado en las últimas décadas y que afectan y siguen afectando a los mismos pobres.

\section{CONCLUSIONES}

En La Ceiba, las manifestaciones de pobreza según el NBI se expresan, entre otras en condiciones insuficientes de las viviendas, acceso a saneamiento básico, educación y hacinamiento. La situación de pobreza es una experiencia cotidiana que se vive en forma diferenciada según la edad, el sexo, la posición de la familia, entre otras variables.

- Las viviendas tienen características variadas en cuanto al tamaño, forma y materiales utilizados para su construcción. Una casa típica que se puede encontrar en La Ceiba es aquella construida con techo de zinc, piso de cemento y paredes de bloque de cemento, aunque en distintas áreas sobre todo en el área rural se puede encontrar viviendas en circunstancias no habitables, ya que no presentan las condiciones óptimas para proteger a la familia que reside en esta. Hay viviendas construidas de bahareque, adobe, palos y hasta de materiales de desecho que no son las propicias para residir pero la pobreza misma hace que las familias opten por conformarse con lo poco que tienen.

\section{Cuadro No.12. Instituciones educativas presentes en La Ceiba}

\begin{tabular}{|c|c|c|c|c|}
\hline \multirow[t]{2}{*}{ Instituciones } & \multicolumn{2}{|c|}{ Publicas } & \multicolumn{2}{|c|}{ Privadas } \\
\hline & Rurales & Urbanas & Rurales & Urbanas \\
\hline \multicolumn{5}{|l|}{ Jardines de Niños } \\
\hline Número de instituciones & 6 & 33 & 0 & 32 \\
\hline Número de docentes & 9 & 58 & 0 & 80 \\
\hline Número de Estudiantes & 234 & 2,082 & 0 & 1,612 \\
\hline \multicolumn{5}{|l|}{ Escuelas primarias } \\
\hline Número de Instituciones & 27 & 39 & 0 & 31 \\
\hline Número de Docentes & 86 & 381 & 0 & 304 \\
\hline Números de Estudiantes & 2,825 & 11,964 & 0 & 5,083 \\
\hline \multicolumn{5}{|c|}{ Centros de Educación Básica General } \\
\hline Número de Instituciones & 2 & 10 & 0 & 0 \\
\hline Número de Docentes & 22 & 222 & 0 & 0 \\
\hline Número de Estudiantes & 499 & 7,351 & 0 & 0 \\
\hline \multicolumn{5}{|l|}{ Institutos Secundarios } \\
\hline Número de Instituciones & 3 & 3 & 1 & 23 \\
\hline Número de Docentes & 34 & 271 & 9 & 523 \\
\hline Número de Estudiantes & 470 & 6,951 & 185 & 7,513 \\
\hline \multicolumn{5}{|l|}{ Instituto a Distancia (ISEMED) } \\
\hline Número de Instituciones & 0 & 1 & 0 & 0 \\
\hline Número de Docentes & 0 & 29 & 0 & 0 \\
\hline Número de Estudiantes & 0 & 624 & 0 & 0 \\
\hline \multicolumn{5}{|l|}{ Escuelas Nocturnas } \\
\hline Número de Instituciones & 0 & 4 & 0 & 0 \\
\hline Número de Docentes & 0 & 18 & 0 & 0 \\
\hline Número de Estudiantes & 0 & 243 & 0 & 0 \\
\hline
\end{tabular}


- El 16.52\% de la población de La Ceiba no está conectada a la tubería de agua potable por diferentes motivos, pero que afecta directamente a la salud sobre todo la de los niños y niñas. Hay muchos casos de personas que no pueden conectarse a la tubería pública porque no cuentan con los recursos necesarios debido a los altos costos de este líquido vital y por consecuencia tienen que recurrir a los ríos, o utilizar agua de pozo para realizar sus actividades diarias.

- Casi la mitad de los hogares ceibeños presenta condiciones de hacinamiento ya que en cada cuarto habitan más de tres personas, esto puede ser ocasionado por varios aspectos: 1 . que en una misma casa vivan dos familias ó más, 2. que las viviendas sean muy pequeñas y 3 . que la familia sea muy numerosa.

- En el municipio de La Ceiba el $87.55 \%$ de la población sabe leer y escribir, hoy en día se ha visto que la educación se está tornando en un aspecto fundamental para la ciudad. La Ceiba cuenta con 37 escuelas oficiales urbanas, 25 escuelas privadas urbanas, 27 escuelas oficiales rurales, 3 institutos secundarios públicos en el área urbana y 3 en el área rural, 23 institutos secundarios privados en el área urbana y 1 en el área rural, existen 3 universidades privadas y 2 públicas que han ampliado las oportunidades a los jóvenes para que sigan sus estudios universitarios. No obstante, la décima parte de la población sigue siendo analfabeta, esto es preocupante debido a que la ciudad de La Ceiba es la tercera ciudad más importante de Honduras; por lo que es necesario crear programas para disminuir este flagelo y así mejorar la educación local y nacional, sin embargo, la calidad misma es cuestionable pero este aspecto tendría que ser analizado con mayor profundidad.

\section{BIBLIOGRAFÍA}

\section{Libros}

- Discusión crítica de tres métodos de medición de la Pobreza UNAH-PLATS 2007.

- Socialización y seguimiento a los ODM objetivo 1 y 2 (paquete educativo básico).

- ERP completo pdf. 2001

- $\quad$ PNUD informe sobre metas del Milenio 2003

- XVI Censo de Poblacional y Vivienda,INE 2001,

Estudios anteriores

- Estudio Diagnóstico La Ceiba, EPYPSA. pdf. 2005

- Estudio Indicadores Ambientales La Ceiba (Aradhos) 2007

- Estudio sobre pobreza crítica en C.A, Román Rafael 1997

Internet

- http://www.centroamericainclusiva.com/DOC_CENTROAM/UD-M2/lb-M2-UD.pdf

- www.comparte.org

- www.ine.com.hn.

- www.luventicus.org/mapas/honduras/atlantida.html

- http://www.undp.un.hn/odm_glosario.htm

- xxxv encuesta permanente de hogares de propósitos múltiples

Instituciones

- Departamento de Investigación y Estadística Municipal de La Ceiba (DIEM)

- Municipalidad de La Ceiba

- Secretaria de Departamental Educación. 